\title{
On the existence of additional streams with deep fronts in brightness rays of the streamer belt
}

\author{
M.V. Eselevich ${ }^{1}$, V.G. Eselevich ${ }^{1}$ \\ ${ }^{1}$ Institute of Solar-Terrestrial Physics, Irkutsk, Russia, email:esel@iszf.irk.ru
}

In Eselevich V. and M. Solar Phys. 195, 319, 2000 is shown that in brightness rays of the streamer belt from time to time there exist additional plasma streams of enhanced density moves antisunward and occupies a separate ray. The duration of additional streams can range from a few hours to several days. For that reason, some of them may be categorized as sporadic SW streams and the others as quasi-stationary SW streams. Preliminary investigations showed that the leading edge of such streams can be extremely small in width. The purpose of this paper is to verify the conclusions about the existence of additional plasma streams with steep fronts in streamer belt rays, based on analyzing the calibrated data from LASCO/SOHO $\mathrm{C} 2$ and $\mathrm{C} 3$.

The technique for deriving quantitative information from white-light corona images involved the following sequence of steps. For each white-light corona image from the $\mathrm{C} 2$ or C3 instrument, we constructed normalized corona brightness distributions $P(\Lambda)$ or $P(R)$, respectively, for a fixed distance $R$ from the Sun's center or the apparent latitude $\Lambda$ separately for the $\mathrm{E}$ or $\mathrm{W}$ limbs. To characterize the brightness of an individual ray we used the profile of the ray brightness $\Pi(\Lambda)=P(\Lambda)-P_{S}(\Lambda)$, the ray brightness amplitudes $P_{R}(\Lambda)=P_{M}(\Lambda)-P_{S}(\Lambda)$, as well as the angular size $d$ (its determination is given in Eselevich V. and M. Solar Phys. 188, 299, 1999). Here $P_{S}(\Lambda)$ is the "background" brightness that represents an averaging of the $P(\Lambda)$-curve over angle $10^{\circ}$ at a given time $t$. To verify the results obtained, and also to derive new information about the physics of the processes under investigation, in this paper we use the time-difference functions of brightness $\Pi\left(\Lambda, t-t_{0}\right)=P(\Lambda, t)-P\left(\Lambda, t_{0}\right)$, where $t_{0}<t$. With such definitions of the ray brightness function $\Pi(t, \Lambda, R)$ and of the time-difference of the brightness function $\Pi\left(\Lambda, t-t_{0}\right)$, the contribution from the F-corona was excluded. That enabled us to determine the average (over the angular diameter $d$ of the ray) value of the plasma density $N_{R}$ at a given distance and a given moment of time without using the polarization brightness data, the main purpose of which is to exclude the influence of the F-corona. As an example let us consider image for June 1-2, 1998. During that time, a small section of the streamer belt located at an apparent latitude of $\Lambda \approx-20^{\circ} \div-30^{\circ}$ near the western limb was almost normal to the plane of the sky. A streamer belt with this orientation can be observed as either one or two rays, which can have different or equal brightness. Figure 4 at the page 693 of paper Eselevich. Astronomy Report. V.48. N.8. 2004 shows the profile $\Pi(\Lambda)$ within the given section for various $R$ and at various times t. We can see now one ray (A) expands and another (B) forms. The time lag between the expansion of ray $\mathrm{A}$ and the formation of ray $\mathrm{B}$ at various distances $\mathrm{R}$ are due to the motion of an additional, steep-fronted plasma stream from the Sun at a latitude of $\Lambda \approx-23^{\circ}$. A twodimensional map of the ray brightness, $\Pi(\Lambda, R)$, in the vicinity of rays $\mathrm{A}$ and $\mathrm{B}$ is shown for several successive times in Figure 1. The arrows mark the positions of the leading front of the additional stream filling ray $\mathrm{B}$ at successive times. The investigations is shown 


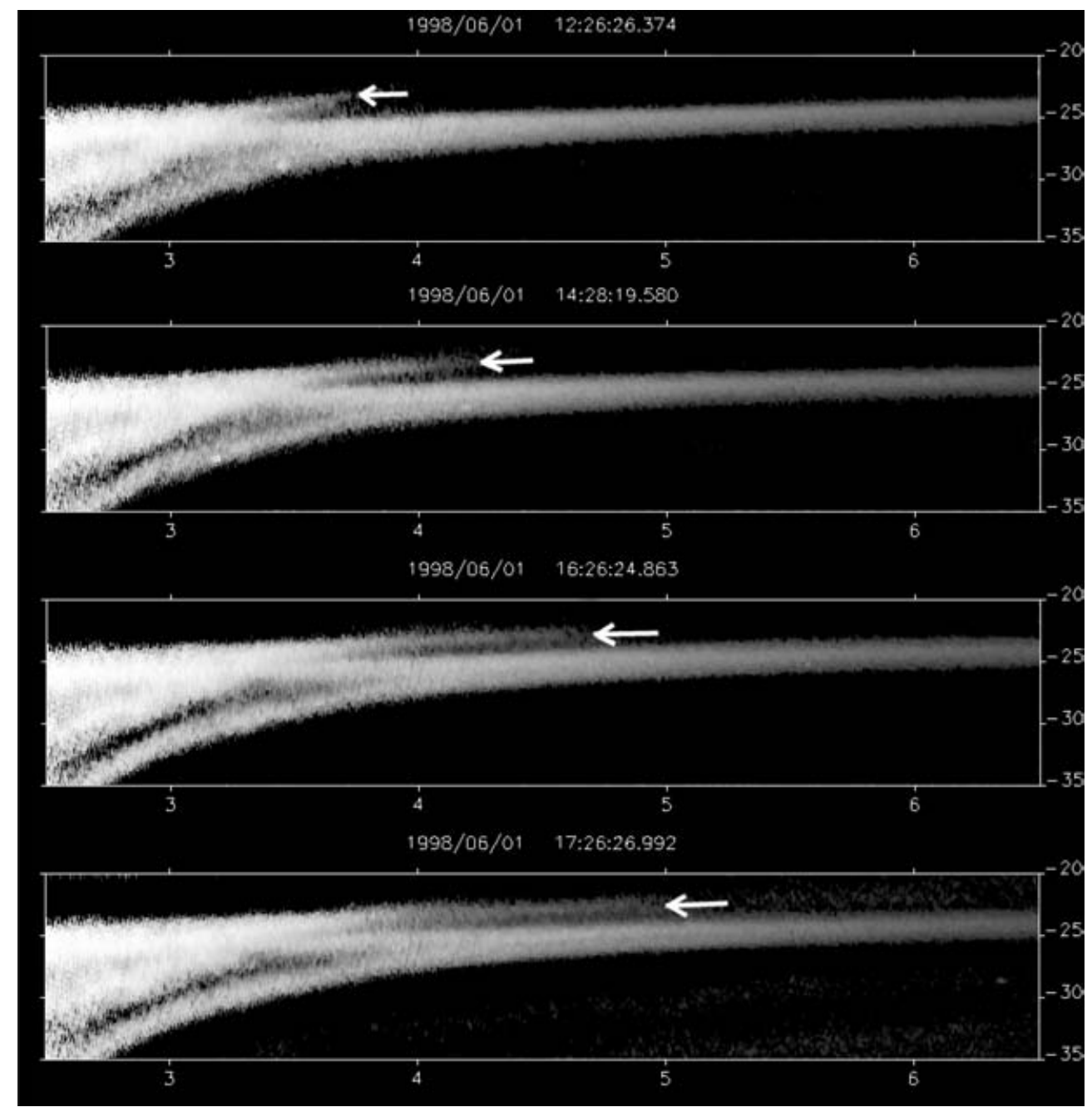

Figure 1. Two-dimensional map of the ray brightness $\Pi(\Lambda, R)$ in the vicinity of rays $\mathrm{A}$ and $\mathrm{B}$ for successive times on June 1,1998. The arrows mark the positions of the additional stream filling ray $\mathrm{B}$ at several times. The lower ray $\mathrm{A}$ is clearly visible during the entire period (W limb; LASCVO/C2 data).

that additiona streams travel with a steep front whose width $d \approx 0.10 R_{\odot}$, on the order of the spatial resolution of the LASCO-C3 instrument. The additional streams are akin to quasi-stationary slow SW streams in the streamer belts in the following quantities: plasma density, directed velocity, and duration of streams. 